\title{
Carvedilol Reestablishes Long-Term Potentiation in a Mouse Model of alzheimer's Disease
}

\author{
Isabel Arrieta-Cruz ${ }^{\mathrm{a}}$, Jun Wang ${ }^{\mathrm{a}}$, Constantine Pavlides ${ }^{\mathrm{b}}$ and Giulio Maria Pasinetti ${ }^{\mathrm{a}, \mathrm{c}, *}$ \\ ${ }^{a}$ Department of Neurology, Mount Sinai School of Medicine, New York, New York, USA \\ ${ }^{\mathrm{b}}$ Rockefeller University, New York, NY, USA \\ ${ }^{\mathrm{c}}$ Geriatrics Research, Education and Clinical Center, James J. Peters Veteran Affairs Medical Center, Bronx, NY, \\ USA
}

Accepted 15 April, 2010

\begin{abstract}
In this study, we examined acute effects of carvedilol, a nonselective $\alpha / \beta$-adrenergic receptor blocker, on neuronal transmission and long-term potentiation (LTP) in six month-old TgCRND8 mice and their wild-type age-matched controls. Field excitatory postsynaptic potentials were recorded in the CA1 region of the hippocampus from carvedilol- or vehicle dimethyl sulfoxide-treated slices, and differences in basal synaptic transmission and LTP were assessed. Carvedilol treatment produced a significant increase in basal synaptic transmission and LTP in TgCRND8 mice, as compared to their vehicle-treated slices, in which basal neuronal transmission and LTP decreased. Interestingly, carvedilol significantly suppressed spontaneous seizure activity in TgCRND8 mice as measured by the number of slices showing epileptic discharges as well as the number of spikes within these and the amplitude of the second spike, measured at baseline and end of recording. In contrast, vehicle-treated slices in TgCRND8 mice did not show a significant decrease in epileptic discharges. These results suggest that carvedilol reestablishes basal synaptic transmission, enhances neuronal plasticity and suppresses neuronal hyperexcitability in TgCRND8 mice.
\end{abstract}

Keywords: Alzheimer's disease, carvedilol, epileptic discharges, hippocampus, LTP, neuronal transmission, synaptic plasticity, TgCRND8 mice

\section{INTRODUCTION}

Recent in vivo studies have suggested that some antihypertensive drugs, including $\beta$-adrenergic blockers, could selectively protect against Alzheimer's disease (AD)-type neuropathology by preventing the accumulation of oligomeric amyloid- $\beta(\mathrm{A} \beta)$ peptides in the brains of $\mathrm{Tg} 2576$ mice, an $\mathrm{AD}$ animal model [1]. Studies performed in primary hippocampal cultures from rats and an $\mathrm{A} \beta \mathrm{PPswe} / \mathrm{PS} 1 \mathrm{AD}$ mouse model have

\footnotetext{
${ }^{*}$ Correspondence to: Giulio Maria Pasinetti, M.D., Ph.D., Department of Neurology, Mount Sinai School of Medicine, 1 Gustave L. Levy Place, Box 1137, New York, NY 10029, USA. Tel.: +1 212241 7938; Fax: +1 212876 9042; E-mail: giulio.pasinetti@mssm.edu.
}

shown that activation of $\beta_{2}$-adrenergic receptors by isoproterenol or clenbuterol, $\left(\beta_{2}\right.$-adrenergic selective agonists), stimulated $\gamma$-secretase activity and accelerated amyloid plaque formation [2].

There is substantial evidence concerning the role of the adrenergic system in epilepsy [3]. The majority of these studies support the view that some adrenergic antagonists produce a strong suppressive effect on seizure activity, involving, in part, $\alpha_{2}$-adrenergic receptors; in contrast, the role of $\beta$-adrenergic receptors on epileptic activity is not well understood [4]. The presence of spontaneous nonconvulsive seizure activity in cortical and hippocampal networks in $\mathrm{A} \beta$-overexpressing mouse models of $\mathrm{AD}$ has been correlated with aberrant excitatory neuronal network activity in vivo, which 
may trigger inhibitory mechanisms in hippocampal circuits, limiting the capacity for synaptic plasticity and contributing to AD-related cognitive dysfunction [5]. To date, there is no information regarding the effects of adrenergic receptor blockers on spontaneous seizure activity in the TgCRND8 AD mouse model.

We have recently shown that, using the TgCRND8 AD mouse model, an aggressive early onset model of brain amyloidosis, chronic treatment with carvedilol (a nonselective $\alpha / \beta$-adrenergic receptor blocker) significantly reduced the oligomerization of $\mathrm{A} \beta$ peptides and the number of $\mathrm{A} \beta$ plaques in the cerebral cortex and hippocampus, which also correlated with improvements in spatial memory [6]. Given that carvedilol attenuated cognitive deterioration in TgCRND8 mice, the present study examined the effects of carvedilol on basal neuronal transmission and long-term potentiation (LTP), as measured by field extracellular recordings in hippocampal slices from TgCRND8 mice.

\section{MATERIALS AND METHODS}

\section{Animals and brain slice preparation}

B6C3F1 wild-type (WT) (Charles River, Wilmington, MA, USA) and TgCRND8 male mice $(n=8-10$ per group), six months old at the time of testing, were housed with food and water available ad libitum, and maintained on a 12:12 h light:dark cycle with lights on at $07: 00 \mathrm{~h}$ in a temperature-controlled $\left(20 \pm 2^{\circ} \mathrm{C}\right)$ room. All procedures and protocols were approved by The Mount Sinai School of Medicine's Institutional Animal Care and Use Committee (IACUC) through The Center for Comparative Medicine and Surgery. Hippocampal brain slices $(350 \mu \mathrm{m})$ were made using a tissue chopper (Mickle Lab Engineering. Co. Ltd, United Kingdom) and placed into oxygenated artificial cerebrospinal fluid (ACSF) at room temperature for a minimum of $2 \mathrm{~h}$ to acclimatize. The composition of ACSF (mM) was: $10 \mathrm{D}$-glucose, $124 \mathrm{NaCl}, 1.25 \mathrm{NaH}_{2} \mathrm{PO}_{4}, 26 \mathrm{NaHCO}_{3}$, $4.9 \mathrm{KCl}, 1 \mathrm{CaCl}_{2}$ and $4 \mathrm{MgCl}_{2}$; and was saturated with $95 \% \mathrm{O}_{2}+5 \% \mathrm{CO}_{2}$. Slices (1 to 2 slices were recorded per animal) were treated for $4 \mathrm{~h}$ with 100,200 , or $400 \mathrm{nM}$ carvedilol in $0.1 \%$ of dimethyl sulfoxide (DMSO) (Microsource Discovery System Inc, CT, USA) or vehicle ( $0.1 \%$ DMSO) (Sigma-Aldrich, MO, USA) in oxygenated ACSF.

\section{Electrophysiological recordings}

Slices were transferred to a recording chamber (Fine Science Tools Inc, CA, USA) and perfused continuously with oxygenated-ACSF at $32^{\circ} \mathrm{C}$. For extracellular recordings, Schaffer collateral projections from the CA3 region were stimulated with a monopolar stainless steel electrode for activation of CA1 neurons. A recording borosilicate glass electrode, filled with $3 \mathrm{M}$ $\mathrm{NaCl}$ with 1-2 $\mathrm{M} \Omega$ resistance was placed in the CA1 region to record field excitatory postsynaptic potentials (fEPSP), see Fig. 2A. Constant current pulses (150 $\mu$ s, $20-30 \mu \mathrm{A})$ were delivered using a stimulus isolation unit (A310 Accupulser, World Precision Instruments, FL, USA) and evoked fEPSPs were recorded with an amplifier (A-M Systems, WA, USA) and monitored on a digital oscilloscope. Responses were elicited every $1 \mathrm{~min}$ and were digitized, stored and analyzed using an Apple Macintosh computer and custom built software based on Lab-View 5.1 software (National Instruments, TX, USA). Basal synaptic transmission was assessed by determining the input/output function of the fEPSP slope and the amplitude of the population spike by a stepwise increase in stimulation intensity between minimum and maximum responses. A test stimulus was chosen at approximately $50 \%$ of the maximum evoked responses. Baseline responses were then recorded for 10 min followed with HFS (4 trains of $100 \mathrm{~Hz}, 1 \mathrm{~s}$ of duration, $20 \mathrm{~s}$ inter-train intervals) to induce LTP, and fEPSPs were monitored for $60 \mathrm{~min}$ to assess the magnitude of potentiation.

\section{Assessment of epileptic discharge activity}

Hippocampal slices that presented more than two spikes in the field potentials were considered epileptic. The number of spikes and amplitude of the second abnormal spike in hippocampal slices from TgCRND8 or WT control mice, with or without acute carvedilol treatment, were measured at baseline and at the end of recordings after induction of LTP.

\section{Statistical analysis}

Data were analyzed using Prism software (V4.03, GraphPad Software, Inc, San Diego, CA). Data were presented as mean \pm SEM and analyzed using a twoway ANOVA in a within-subjects design, followed by Bonferroni's post hoc tests for pair-wise comparisons. Significant differences were set to ${ }^{*} p<0.05,{ }^{* * *} p<$ 0.01 and $^{* * *} p<0.001$ or $\# \# \# p<0.001$. 
Table 1

Epileptic discharges in hippocampal slices from 6 months old mice treated with carvedilol

\begin{tabular}{ccc}
\hline Mice & Vehicle & Carvedilol \\
\hline WT & $1(17 \%)$ & $2(33 \%)$ \\
& $n=6$ slices & $n=6$ slices \\
TgCRND8 & $9(90 \%)$ & $5(41 \%)$ \\
& $n=10$ slices & $n=12$ slices \\
\hline
\end{tabular}

\section{RESULTS}

A carvedilol dose-response curve (100, 200, and $400 \mathrm{nM}$ ) was performed in hippocampal slices from WT mice to measure its effects on LTP, to select the most appropriate dose for this study. We observed that $200 \mathrm{nM}$ carvedilol produced significantly higher LTP than vehicle, 100, or $400 \mathrm{nM}$ carvedilol, measured by the fEPSP slope and the amplitude of population spike (Fig. 2B). The rest of the studies were, therefore, run at this dose.

We observed that $90 \%$ of vehicle-treated slices and $41 \%$ of carvedilol-treated slices from TgCRND8 mice presented epileptic discharges in the action field potentials (Fig. 1A and Table 1). In contrast, between $17 \%$ and $33 \%$ of vehicle- or carvedilol-treated slices from WT mice showed an epileptic pattern (Fig. 1B and Table 1); however, these differences were not significant (Fisher's test; $p>0.05$ ). Carvedilol significantly reduced the number of abnormal spikes and decreased the amplitude of the second abnormal spike at baseline and the end of recording, compared to vehicletreated slices, in TgCRND8 mice (Table 2). In WT mice, carvedilol did not modify the number of spikes or the amplitude of the second spike (Table 2).

We observed significant differences in basal synaptic transmission between TgCRND8 and WT mice. For the TgCRND8 group ( $n=9$ slices per group), in vehicle-treated slices the maximum (mean \pm SEM) fEPSP slope was $3.4 \pm 0.7 \mathrm{mV}$ (Fig. 2C) and the amplitude of the population spike was $3.8 \pm 0.7 \mathrm{mV}$ (Fig. 2E) whereas in carvedilol-treated slices the maximum the fEPSP slope was $4.31 \pm 0.62 \mathrm{mV}$ (Fig. 2C) and the amplitude of the population spike was $5.16 \pm 0.71 \mathrm{mV}$ (Fig. 2E), indicating that acute carvedilol treatment restores basal synaptic transmission in these mice. In contrast, for the WT group ( $n=5$ slices per group), there were no significant differences in the maximum fEPSP slope and the amplitude of the population spike when comparing vehicle and carvedilol (Fig. 2C and 2E).

We observed a significant increase in LTP in carvedilol-treated versus vehicle-treated slices from
A)
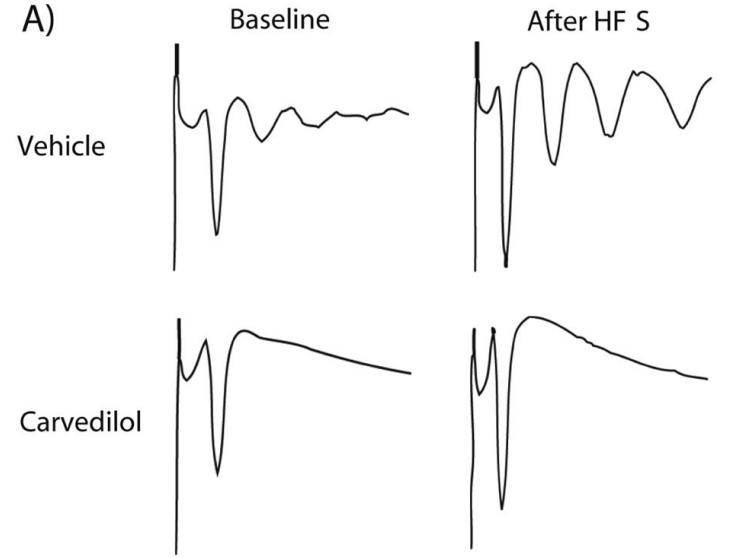

B)
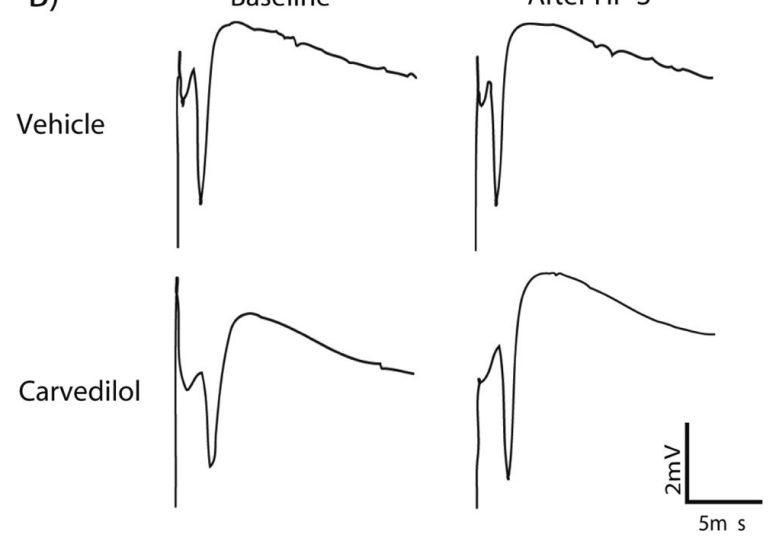

Fig. 1. Representative field potentials in hippocampal slices from 6-month old TgCRND8 mice (A) and their age-matched WT controls (B). Potentials were obtained in the CA1 region with stimulation of the Schaffer collaterals. $90 \%$ of vehicle-treated slices from TgCRND8 mice (A) showed epileptic discharges which became of higher amplitude and number after induction of LTP. In contrast, the carvedilol-treated slices showed a normal pattern both at the beginning of recording (baseline) and following high-frequency stimulation. In the vehicle-treated DMSO and carvedilol-treated slices from WT mice (B), between $17-33 \%$ of all slices presented epileptic discharges. (See Table 1).

TgCRND8 mice, as measured by both the fEPSP slope (Fig. 2D) and population spike (Fig. 2F). These differences between vehicle- and carvedilol-treated slices from TgCRND8 mice were evident in the last $10 \mathrm{~min}$ of recording for the fEPSP slope $(106.28 \pm 10.44 \%$ for vehicle vs. $264.92 \pm 11.10 \%$ for carvedilol; ${ }^{* * *} p<$ 0.001 , Fig. 2D top panel) and the population spike $(117.68 \pm 5.74 \%$ for vehicle versus $191.74 \pm 14.12 \%$ for carvedilol; ${ }^{* * *} p<0.001$, Fig. $2 \mathrm{~F}$ top panel). In contrast, we did not observe significant differences in LTP between vehicle- and carvedilol-treated slices from WT mice. 
Table 2

Number of spikes and amplitude of the second abnormal spike in hippocampal slices from 6 months old mice treated with carvedilol. Data represent mean \pm SEM and were analyzed using a Two-Way ANOVA followed by Bonferroni's post hoc tests for pair-wise comparisons. Significant differences were set to ${ }^{*} p<$ 0.05 between carvedilol group versus vehicle group

\begin{tabular}{ccccc}
\hline Mice & Treatment & $\begin{array}{c}\text { Number of spikes } \\
\text { (Mean } \pm \text { SEM) }\end{array}$ & $\begin{array}{c}\text { Amplitude }(\mathrm{mV}) \text { of } \\
\text { the 2nd spike } \\
\text { at baseline } \\
\text { (Mean } \pm \text { SEM) }\end{array}$ & $\begin{array}{c}\text { Amplitude }(\mathrm{mV}) \\
\text { of the 2nd spike at end } \\
\text { of recording } \\
\text { (Mean } \pm \text { SEM) }\end{array}$ \\
\hline WT & Vehicle & $1.16 \pm 0.16(n=6)$ & $0.54(n=1)$ & $0.81(n=1)$ \\
TgCRND8 & Carvedilol & $1.66 \pm 0.49(n=6)$ & $1.29 \pm 0.02(n=2)$ & $1.95 \pm 0.28(n=2)$ \\
& Vehicle & $2.80 \pm 0.26(n=10)$ & $0.98 \pm 0.14(n=8)$ & $1.60 \pm 0.19(n=8)$ \\
& Carvedilol & $1.66 \pm 0.25^{*}(n=12)$ & $0.54 \pm 0.9^{*}(n=5)$ & $0.89 \pm 0.28(n=5)$ \\
\hline
\end{tabular}

\section{DISCUSSION}

The key findings from the present study are that acute carvedilol treatment of hippocampal slices from TgCRND8 mice reestablished basal synaptic transmission, improved LTP and dramatically suppressed neuronal hyperexcitability as measured by epileptic discharge activity. This is the first study directly testing the effects of a non-selective $\alpha / \beta$-adrenergic blocker, specifically carvedilol, on synaptic transmission and plasticity in a mouse model of AD.

Studies in $\mathrm{A} \beta$-overexpressing mice have reported alterations in synaptic function. Directly related to the present work, some studies have shown suppression in basal synaptic transmission and a severe impairment in LTP from TgCRND8 mice [7-9]. Our findings confirm and extend these previous reports regarding synaptic dysfunction in the hippocampus from TgCRND8 mice, which appears to have a strong correlation with progressive cognitive impairment in this mouse model of AD [9].

Data obtained recently by our team revealed that the abnormalities in learning and memory observed in TgCRND8 mice may be due to the contribution of a number of factors, including the toxic effects of $\mathrm{A} \beta$ oligomers and a reduction in thin spine density in neurons of the cerebral cortex of these animals, which may be reversed by carvedilol (Pasinetti et al., unpublished observation). The mechanisms by which carvedilol may improve learning and memory in TgCRND8 mice are not well understood. However, they could be explained, in part, by the reestablishment of normal levels of basal synaptic transmission and LTP in the hippocampus of these mice, as shown in the present study. A recent study found that activation of $\beta_{2}$-adrenergic receptors stimulates $\gamma$-secretase activity and accelerates amyloid plaque formation; these effects could be mediated through the guanine nucleotide-binding proteins activating intracellular second messengers, such as cAMP, which is crucial for receptor desensitization and subsequent trafficking of $\gamma$-secretase to late endosomes and lysosomes increasing $\mathrm{A} \beta$ production [2]. Our findings on the effects of a $\beta$-adrenergic receptor antagonist on LTP in TgCRND8 mice are in contrast to that seen in normal mice, where for example, the isoproterenol, a selective agonist, induces LTP and propranolol, a nonselective antagonist, suppresses LTP in the hippocampus. This modulation seen in normal mice is crucial for the initiation of long-term memory storage and memory retrieval $[10,11]$. These differences in effects could be explained in several ways, including: 1) selectivity of adrenergic receptors; 2) application of the drug; and 3) timing of treatment. In support of our findings, a recent study showed that inhibition of LTP by exogenous $\mathrm{A} \beta$ is prevented through modulation of $\beta_{2}$-adrenergic receptors in hippocampal slices of normal rats [12]. Our results provide novel evidence that carvedilol may attenuate the toxicity of $\mathrm{A} \beta$ peptides and contribute to a better synaptic transmission and neuronal plasticity in the hippocampus from TgCRND8 mice.

Moreover, in the present study, $90 \%$ of vehicle treated slices from TgCRND8 mice presented spontaneous seizure activity, which may contribute to the synaptic dysfunction observed in these mice. Interestingly, carvedilol suppressed spontaneous seizure activity, and improved synaptic transmission and LTP. Pharmacological studies using specific agonists/antagonists of $\alpha$ - or $\beta$-adrenergic receptors have provided evidence that modulating the activity of $\alpha$-adrenergic receptors have anticonvulsive effects in normal mice [4], which supports the notion that carvedilol is blocking, at least in part, the activation of $\alpha$-adrenergic receptors, resulting in a decrease in spontaneous seizure activity in TgCRND8 mice. In some transgenic mice, $\beta_{1}$ adrenergic receptor over expression can produce spontaneous seizures, which can be suppressed by their antagonists [13]. On other hand, studies in nonneu- 
A)

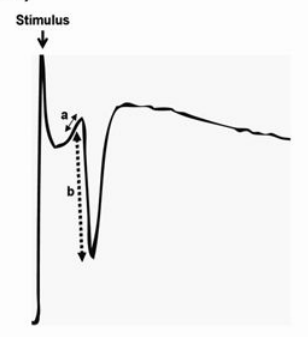

C)

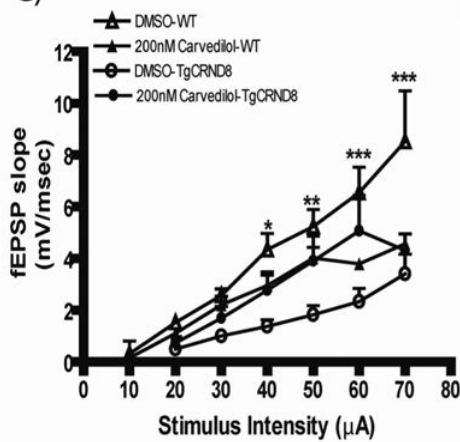

E)

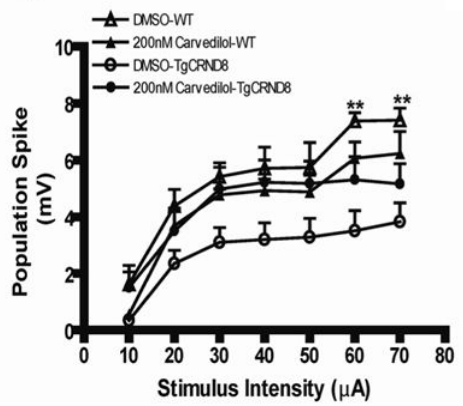

B)

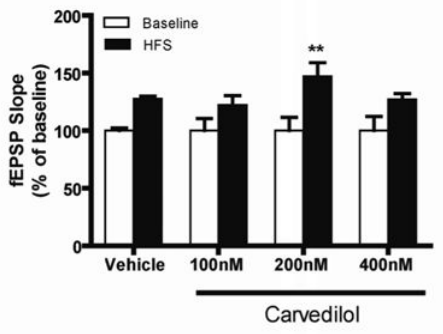

D)

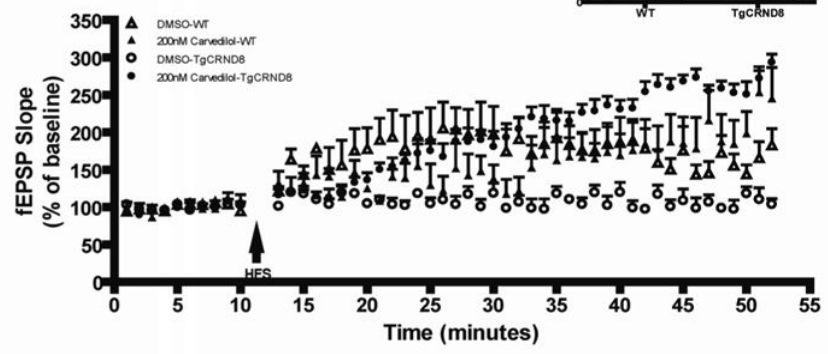

F)

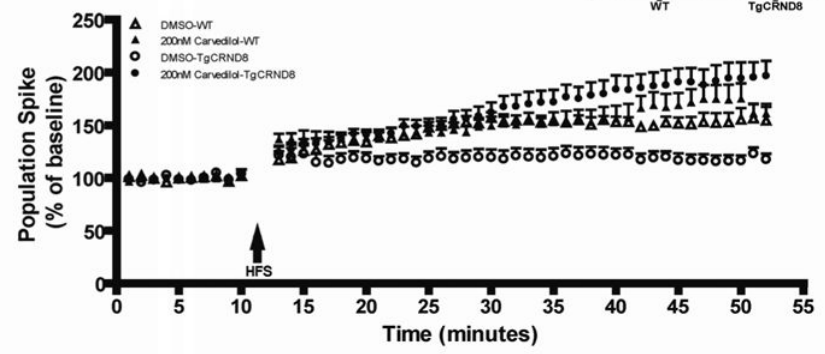

Fig. 2. Effects of carvedilol on basal synaptic transmission and LTP in hippocampal slices from 6-month-old TgCRND8 and WT control mice. Measurements of the fEPSP slope (a) and amplitude of population spike (b) were performed as shown on schematic (A). The data represent mean \pm S.E.M. and were analyzed using a 2-way ANOVA. Significant differences were designated as follows: ${ }^{*} p<0.05 ;{ }^{* *} p<0.01$; and ${ }^{* * *} p<$ 0.001 or \#\#\# $p<0.001$. A carvedilol dose-response curve was performed in WT control mice; a significant increase of LTP was observed using $200 \mathrm{nM}$ carvedilol for fEPSP slope (B, left panel) and the amplitude of the population spike (B, right panel). For basal synaptic transmission, in vehicle-treated DMSO slices from TgCRND8 mice $(\bigcirc, n=9$ slices per group) a significant reduction in basal synaptic transmission was seen compared to the WT group $\left(\Delta, n=5\right.$ slices per group) for fEPSP slope $\left({ }^{* * *} p<0.001, \mathrm{C}\right)$ and the maximum amplitude of the population spike $\left({ }^{* *} p<0.01, \mathrm{E}\right)$. In contrast, in carvedilol-treated slices there were no significant differences in fEPSP slope (C) or the maximum amplitude of the population spike (E) when comparing TgCRND8 (O) vs. WT mice $(\boldsymbol{\Lambda})$. For LTP studies, the values of fEPSP slope (D) and of the amplitude the population spike $(\mathrm{F})$ are expressed as percentages compared to their own baselines normalized to $100 \%$. The arrow indicates time of tetanus to induce LTP. The top panel of each graph shows the mean \pm S.E.M. of the last $10 \mathrm{~min}$ of recording after tetanus. The results show a significant increase in LTP of the carvedilol-treated slices compared to vehicle-treated DMSO slices from TgCRND8 mice in both fEPSP slope (D, top panel; $\left.{ }^{* * *} p<0.001\right)$ and the amplitude of the population spike (F, top panel; $\left.{ }^{* * *} p<0.01\right)$. In contrast, in slices from WT mice, there were no significant differences between carvedilol and vehicle-treated DMSO slices, as measured by either the fEPSP slope or the population spike. Interestingly, in vehicle-treated DMSO slices, TgCRND8 mice showed a significant reduction in LTP compared to WT mice in both fEPSP slope (D, top panel; $\# \# \# p<0.001$ ) and the amplitude of the population spike (F, top panel; \#\#\# $p<0.001$ ). 
ronal cells, specifically in rabbit ventricular myocytes, carvedilol blocked $\mathrm{K}^{+}$and L-type $\mathrm{Ca}^{2+}$ currents, tested by whole-cell patch clamp, showing a decrease in the spontaneous firing frequency, depolarization of the maximal diastolic potential and prolongation of the action potential duration [14], suggesting that a selective blockade of these channels might have similar effects on neuronal cells. It has been shown that calcium channel antagonists block epileptiform activity in adult rat hippocampal slices, suggesting an essential role of Ltype $\mathrm{Ca}^{2+}$ channel in epileptogenesis [15]. Although the role of $\beta$-adrenergic receptors on epileptic activity in $\mathrm{AD}$ is not fully understood, our findings shed new light on their underlying electrophysiological mechanisms in AD experimental models, and provide impetus for the further evaluation of carvedilol in AD.

\section{ACKNOWLEDGMENTS}

These studies were generously supported by funding from the Altschul Foundation, the National Institute of Aging (U01 AG02219), and the Geriatrics, Research, Education and Clinical Center at the James J. Peters Veteran Affairs Medical Center awarded to GMP. We would like to thank Dr. Bing Gong for his comments and Miss Hayley Fivecoat for her assistance in the preparation of this manuscript.

Authors' disclosures available online (http://www.jalz.com/disclosures/view.php?id=413).

\section{REFERENCES}

[1] Wang J, Ho L, Chen L, Zhao Z, Zhao W, Qian X, Humala N, Seror I, Bartholomew S, Rosendorff C, Pasinetti GM (2007) Valsartan lowers brain beta-amyloid protein levels and improves spatial learning in a mouse model of Alzheimer disease. J Clin Invest 117, 3393-3402.

[2] Ni Y, Zhao X, Bao G, Zou L, Teng L, Wang Z, Song M, Xiong J, Bai Y, Pei G (2006) Activation of beta2-adrenergic receptor stimulates gamma-secretase activity and accelerates amyloid plaque formation. Nat Med 12, 1390-1396.

[3] Giorgi FS, Pizzanelli C, Biagioni F, Murri L, Fornai F (2004)
The role of norepinephrine in epilepsy: from the bench to the bedside. Neurosci Biobehav Rev 28, 507-524.

[4] Stringer JL, Lothman EW (1991) Cholinergic and adrenergic agents modify the initiation and termination of epileptic discharges in the dentate gyrus. Neuropharmacol 30, 59-65.

[5] Palop JJ, Chin J, Roberson ED, Wang J, Thwin MT, Bien-Ly N, Yoo J, Ho KO, Yu GQ, Kreitzer A, Finkbeiner S, Noebels JL, Mucke L (2007) Aberrant excitatory neuronal activity and compensatory remodeling of inhibitory hippocampal circuits in mouse models of Alzheimer's disease. Neuron 55, 697-711.

[6] Wang J, Ono K, Dickstein DL, Arrieta-Cruz I, Zhao W, Qian X, Lamparello A, Subnani R, Ferruzzi M, Pavlides C, Ho L, Hof PR, Teplow DB, Pasinetti GM (2010) Carvedilol as a potential novel agent for the treatment of Alzheimer's disease. Neurobiol Aging, in press.

[7] Chapman PF, White GL, Jones MW, Cooper-Blacketer D, Marshall VJ, Irizarry M, Younkin L, Good MA, Bliss TV, Hyman BT, Younkin SG, Hsiao KK (1999) Impaired synaptic plasticity and learning in aged amyloid precursor protein transgenic mice. Nat Neurosci 2, 271-276.

[8] Ye H, Jalini S, Mylvaganam S, Carlen P (2010) Activation of large-conductance $\mathrm{Ca}(2+)$-activated $\mathrm{K}(+)$ channels depresses basal synaptic transmission in the hippocampal CA1 area in APP (swe/ind) TgCRND8 mice. Neurobiol Aging 31, 591-604.

[9] Bellucci A, Luccarini I, Scali C, Prosperi C, Giovannini MG, Pepeu G, Casamenti F (2006) Cholinergic dysfunction, neuronal damage and axonal loss in TgCRND8 mice. Neurobiol Dis 23, 260-272.

[10] Gelinas JN, Nguyen PV (2005) Beta-adrenergic receptor activation facilitates induction of a protein synthesis-dependent late phase of long-term potentiation. J Neurosci 25, 32943303.

[11] Gelinas JN, Tenorio G, Lemon N, Abel T, Nguyen PV (2008) Beta-adrenergic receptor activation during distinct patterns of stimulation critically modulates the PKA-dependence of LTP in the mouse hippocampus. Learn Mem 15, 281-289.

[12] Wang QW, Rowan MJ, Anwyl R (2009) Inhibition of LTP by beta-amyloid is prevented by activation of beta2 adrenoceptors and stimulation of the CAMP/PKA signalling pathway. Neurobiol Aging 30, 1608-1613.

[13] Kunieda T, Zuscik MJ, Boongird A, Perez DM, Luders HO, Najm IM (2002) Systemic overexpression of the alpha 1Badrenergic receptor in mice: an animal model of epilepsy. Epilepsia 43, 1324-1329.

[14] Cheng J, Niwa R, Kamiya K, Toyana J, Kodama I (1999) Carvedilol blocks $\mathrm{K}^{+}$and L-type $\mathrm{Ca}^{2+}$ currents in rabbit ventricular myocytes. Eur J Pharmacol 376, 189-201.

[15] Kohling R, Straub H, Speckmann EJ (2000) Differential involment of L-type calcium channels in epileptogenesis of rat hippocampal slices during ontogenesis. Neurobiol Dis 7, 471482. 